\section{Influência da reabilitação física sobre aspectos funcionais em indivíduos submetidos à artroplastia total de quadril: uma revisão sistemática}

\author{
Influence of physical rehabilitation on functional aspects in individuals submitted to total hip \\ arthroplasty: a systematic review
}

\section{Resumo}

Objetivo: Avaliar, por meio de revisão sistemática, a influência do tratamento fisioterapêutico sobre a funcionalidade, amplitude de movimento articular e força muscular em pacientes submetidos à artroplastia total de quadril devido à osteoartrite. Métodos: A busca sistemática de ensaios clínicos randomizados e não randomizados foi conduzida utilizando os bancos de dados eletrônicos PubMed, Web of Science, PEDro, Cochrane, Clinical Trials e SciELO, com base nas estratégias de pesquisa recomendadas pelos itens de relatórios preferenciais para análises sistemáticas e metanálises (PRISMA). Resultados: Em geral, protocolos supervisionados por fisioterapeutas associados à realização de exercícios ativos da musculatura periarticular do quadril e de extensores de joelho têm propiciado importante prognóstico físico e funcional. Exercícios resistidos (dinâmicos e isométricos) de alta intensidade são mais eficazes para a funcionalidade. Para a força muscular e Amplitude de movimento (ADM), exercícios dinâmicos com 3 a 5 séries de 8 a 12 repetições com baixa e alta intensidade promoveram ganhos mais expressivos em relação a outras modalidades terapêuticas. Conclusões: As técnicas e os protocolos utilizados pela fisioterapia para o tratamento da ATQ são variados e possuem importante eficácia clínica comprovada na literatura.

Abstract

Objective: by performing a systematic review, the present study aimed to evaluate the influence of physical rehabilitation on functionality, range of motion and musculoskeletal strength in patients submitted to total hip arthroplasty due to osteoarthritis. Methods: a systematic

\footnotetext{
Universidade Federal de Mato Grosso do Sul, Grupo de Pesquisa "Estudos Avançados em Ciências do Movimento e Reabilitação”. Campo Grande, MS, Brasil.

2 Universidade Federal de Mato Grosso do Sul, Laboratório de Estudo do Músculo Estriado (LEME), Programa de Pós-Graduação em Saúde e Desenvolvimento na Região Centro-Oeste. Campo Grande, MS, Brasil.
}

Financiamento da pesquisa: Universidade Federal de Mato Grosso do Sul (UFMS / MEC - Brasil), e Coordenação de Aperfeiçoamento de Pessoal de Nível Superior - Brasil (CAPES) - Código de Financiamento 001.

Os autores declaram não haver conflito na concepção deste trabalho.
Palavras-Chave: Artroplastia de Quadril. Osteoartrite. Exercício. Envelhecimento. 
search for randomized and non-randomized controlled trials was conducted using the PubMed, Web of Science, PEDro, Cochrane, Clinical Trials and SciELO electronic databases, using the search strategies recommended by the Preferred Reporting Items for Systematic Reviews and Meta-Analyses (PRISMA). Results: in general, protocols supervised by physiotherapists associated with performing active exercises of the hip periarticular muscles and knee extensors have provided effective functional prognosis. High intensity resistance exercises (dynamic and isometric) are more effective in improving functionality. Dynamic exercises with three to five sets of eight to twelve low and high intensity repetitions promoted more pronounced increases in muscle strength and range of motion than other

Keywords: Arthroplasty, Replacement, Hip. Osteoarthritis. Exercise. Aging. treatment after THA are wide-ranging and their clinical efficacy is demonstrated in literature.

\section{INTRODUÇÃO}

O envelhecimento populacional tornou-se uma realidade consolidada em vários países ${ }^{1}$. Esse fato tem tido enorme impacto na organização dos sistemas de saúde, pois os idosos são mais susceptíveis a doenças e agravos crônicos ${ }^{2}$.

Neste cenário, a osteoartrite (OA) destaca-se como uma das principais causas de incapacidade funcional em idosos ${ }^{3}$. Essa afecção é caracterizada por alterações estruturais, de grau leve a severo, da cartilagem de articulações sinoviais ${ }^{3}$. Entre as causas da OA, incluem-se fatores biomecânicos, genéticos, sexuais, obesidade, idade e fatores metabólicos ${ }^{4}$. Clinicamente, a OA repercute em dor, rigidez de curta duração, crepitação, diminuição ou perda da função articular, atrofia muscular e também deformidades ${ }^{3}$.

As articulações que suportam carga, como joelho e quadril, são frequentemente afetadas pela $\mathrm{OA}^{3}$. A osteoartrite do quadril é uma das formas mais incapacitantes desta doença ${ }^{4}$. Atualmente, os tratamentos cirúrgicos são indicados para os pacientes com OA que não obtiveram resultados satisfatórios com o tratamento conservador e que apresentam dor, perda da funcionalidade e inabilidade para realizar as atividades de vida diária ${ }^{5}$.

Nesse sentido, a OA configura a condição clínica com maior frequência de indicação para artroplastia total do quadril (ATQ). Embora seja um procedimento radical, a ATQ proporciona melhora da qualidade de vida e retorno precoce às atividades de vida diária ${ }^{5}$. Levando-se em consideração a repercussão clínica e funcional da OA e da ATQ, a fisioterapia tem se mostrado de extrema importância para esses pacientes e visa ao ganho de amplitude de movimento, redução das complicações causadas pelo procedimento cirúrgico, retorno prévio para as atividades, melhora da dor e de deficit funcionais ${ }^{6,7}$.

Estudos têm demonstrado que pacientes que participam de algum tipo de intervenção fisioterapêutica após ATQ alcançam maior recuperação da função física e melhora precoce da qualidade de vida, quando comparados aos que não realizam ${ }^{6,8-18}$. Embora a fisioterapia disponha de diversos recursos terapêuticos para a reabilitação pós-cirúrgica desses indivíduos, as informações quanto à eficácia de protocolos de tratamento ainda são incipientes.

Portanto, torna-se necessária a sistematização de evidências na busca de suporte científico sobre métodos fisioterapêuticos adequados para a reabilitação funcional de pacientes submetidos à ATQ. Com base na literatura existente, tem-se por objetivo descrever os efeitos da reabilitação física sobre funcionalidade, força muscular e amplitude de movimento articular em sujeitos submetidos à ATQ devido a OA.

\section{MÉTODOS}

Este estudo corresponde a uma revisão sistemática da literatura, considerando como critérios de inclusão ensaios clínicos randomizados e não randomizados que integrassem protocolos com exercícios físicos e/ou eletroterapia para o tratamento de indivíduos (homens e mulheres) submetidos à ATQ devido a OA, em comparação a outras formas de intervenção ou que tivessem um grupo controle, e tivessem sido, publicados em revistas cientificas entre janeiro de 1980 e dezembro de 2019. Foram excluídos estudos 
que não contemplassem os critérios de inclusão, revisões sistemáticas, estudo de casos, séries de casos, retrospectivos, estudos observacionais, estudos pilotos e modelos experimentais com animais.

A busca sistemática de ensaios clínicos randomizados e não randomizados foi realizada por meio das bases eletrônicas de dados PubMed, PEDro, Web of Science, Cochrane, Clinical Trials e SciELO, sendo baseada nas estratégias de pesquisa recomendadas pelos itens de Relatórios Preferenciais para Análises Sistemáticas e Metánalise (PRISMA). A busca também foi realizada por meio das citações dos estudos incluídos na revisão. A pesquisa foi executada usando as seguintes combinações de descritores do Medical Subject Heading Terms (MeSH): Exercise, Hip and Arthroplasty; Physical Therapy, Hip and Arthroplasty; Physical Activity, Hip and Arthroplasty; Exercise Therapy, Hip and Arthroplasty.

A pesquisa foi conduzida em dezembro de 2019. Primeiramente, os títulos e resumos de todos os artigos identificados pela estratégia de busca foram avaliados independentemente e em duplicata por dois avaliadores. Todos os resumos que não forneciam informações suficientes sobre os critérios de inclusão e exclusão foram selecionados para leitura na íntegra. $\mathrm{Na}$ segunda etapa, os mesmos revisores avaliaram artigos completos, independentemente e em duplicata, para selecionar aqueles em conformidade com os critérios de elegibilidade. As diferenças entre os revisores foram resolvidas por consenso entre os pesquisadores.

Os avaliadores realizaram a extração de dados de forma independente, mediante utilização de formulários padronizados, que incluíram informações sobre autor e ano de publicação, participantes (número de indivíduos, idade e sexo), desenho do estudo, escala de avaliação, duração do estudo e resultados da intervenção. Na vigência de inconsistência, os documentos originais eram recuperados e investigados em conjunto para definição consensual. Os desfechos de interesse foram: força muscular; amplitude de movimento (ADM); funcionalidade.

O risco de viés na avaliação e metodologia foi analisado pelos mesmos revisores independentes e em duplicata, utilizando a escala de risco de viés $J_{A D A D}{ }^{24}$. Para cada resultado específico, a qualidade da evidência foi baseada em cinco fatores: 1 - descrito como randomizado, 2 - descrito como duplo-cego, 3 - descrição de perdas amostrais, 4 - randomização apropriada e 5 - mascaramento apropriado. Os resultados da escala JADAD são divididos em dois níveis de evidência que classifica o estudo como de má qualidade (pontuação de 0 a 2) ou de alta qualidade (pontuação de 3 a 5 ) $^{19}$.

\section{RESULTADOS}

Foram encontrados 5702 estudos por meio de busca eletrônica. Dentre estes, 5264 trabalhos foram excluídos, por não se enquadrarem nos critérios pré-estabelecidos de elegibilidade. Portanto, 438 foram selecionados para análise detalhada, partindose dos títulos; 321 foram excluídos em razão de duplicidade. Assim, 117 resumos foram avaliados e, destes, 57 foram selecionados para leitura na íntegra. Após as avaliações de texto completo, 26 artigos foram excluídos por não contemplarem os critérios de elegibilidade. Deste modo, 31 estudos foram incluídos na revisão sistemática. Após análise dos estudos citados pelos 31 trabalhos incluídos na revisão, um estudo que não havia sido identificado anteriormente foi incluído nesta revisão. Portanto, 32 estudos fizeram parte desta revisão sistemática.

A Figura 1 mostra as etapas realizadas no processo de seleção dos estudos e as razões para a exclusão. $\mathrm{Na}$ Tabela 1, estão descritas as características dos estudos incluídos nesta revisão, apresentando os seguintes itens: autor, ano de publicação, desenho do estudo, sujeitos do estudo, divisão dos grupos, variáveis analisadas e resultados.

\section{Riscos de viés}

Em relação ao risco de viés dos estudos incluídos na presente revisão sistemática, 29 (90,62\%) estudos foram submetidos à randomização, apenas dois trabalhos (6,25\%) documentaram ser duplo cego e $29(90,62 \%)$ relataram as perdas amostrais (Tabela 2).

De acordo com a pontuação JADAD, somente um trabalho ${ }^{20}$ recebeu pontuação máxima e dois artigos não pontuaram ${ }^{21,22}$. As classificações dos demais estudos estão descritos na Tabela 2 . 


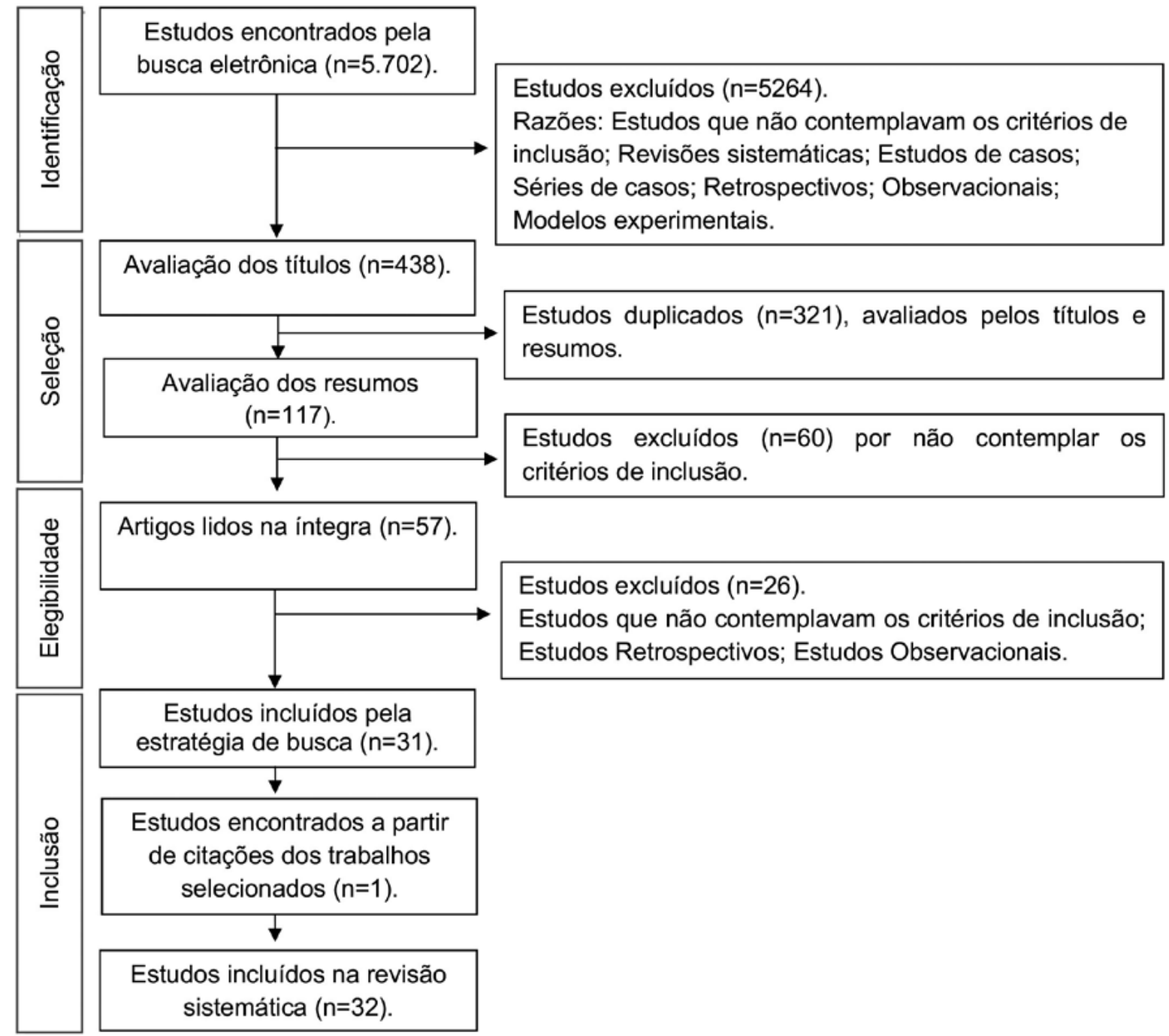

Figura 1. Fluxograma do processo de busca, etapas de seleção e motivos de exclusão dos estudos selecionados para a revisão sistemática. Campo Grande, MS, 2019.

Tabela 1. Características e resultados dos ensaios clínicos randomizados e não randomizados incluídos na revisão sistemática. Campo Grande, MS, 2019.

\begin{tabular}{|c|c|c|c|c|c|}
\hline Autor, Ano & $\begin{array}{l}\text { Desenho do } \\
\text { estudo e sujeitos }\end{array}$ & $\begin{array}{l}\text { Grupo Intervenção } \\
\text { (GI) }\end{array}$ & $\begin{array}{l}\text { Grupo Controle } \\
\text { (GC) }\end{array}$ & Variáveis estudadas & Resultados \\
\hline $\begin{array}{l}\text { Gremeaux et } \\
\text { al., } 2008^{8}\end{array}$ & $\begin{array}{l}\text { Ensaio Clínico } \\
\text { Randomizado } \\
\mathrm{n}=29\end{array}$ & $\begin{array}{l}\text { Grupo LFEMS } \\
\text { (Estimulação elétrica } \\
\text { de baixa frequência e } \\
\text { Fisioterapia) } \\
(\mathrm{n}=16)\end{array}$ & $\begin{array}{l}\text { Fisioterapia } \\
\text { isolada } \\
(\mathrm{n}=13)\end{array}$ & $\begin{array}{l}\text { Força muscular } \\
\text { isométrica máxima } \\
\text { dos extensores do } \\
\text { joelho (dinamômetro } \\
\text { isocinético) e } \\
\text { funcionalidade (FIM). }\end{array}$ & $\begin{array}{l}\text { O GI apresentou melhor } \\
\text { resultado para força } \\
\text { extensora do joelho } \\
(p<0,05) \text { e funcionalidade } \\
(p<0,05) \text {, em comparação } \\
\text { ao GC. }\end{array}$ \\
\hline $\begin{array}{l}\text { Liebs et al., } \\
2012^{13}\end{array}$ & $\begin{array}{l}\text { Ensaio Clínico } \\
\text { Randomizado } \\
\text { Multicêntrico } \\
\mathrm{n}=271\end{array}$ & $\begin{array}{l}\text { Grupo terapia aquática } \\
\text { precoce } \\
\text { (Terapia aquática a } \\
\text { partir do } 6^{\circ} \text { dia de pós- } \\
\text { operatório) } \\
(\mathrm{n}=129)\end{array}$ & $\begin{array}{l}\text { Grupo terapia } \\
\text { aquática após } \\
\text { retirada de } \\
\text { pontos } \\
(\mathrm{n}=142)\end{array}$ & $\begin{array}{l}\text { Funcionalidade } \\
\text { (WOMAC e Lequesne). }\end{array}$ & $\begin{array}{l}\text { O GI alcançou } \\
\text { melhor resultado para } \\
\text { funcionalidade }(p=0,01) \text {. }\end{array}$ \\
\hline
\end{tabular}


Continuação da Tabela 1

\begin{tabular}{|c|c|c|c|c|c|}
\hline Autor, Ano & $\begin{array}{l}\text { Desenho do } \\
\text { estudo e sujeitos }\end{array}$ & $\begin{array}{l}\text { Grupo Intervenção } \\
(\mathrm{GI})\end{array}$ & $\begin{array}{l}\text { Grupo Controle } \\
\text { (GC) }\end{array}$ & Variáveis estudadas & Resultados \\
\hline $\begin{array}{l}\text { Hesse et al., } \\
2003^{14}\end{array}$ & $\begin{array}{l}\text { Ensaio Clínico } \\
\text { Randomizado } \\
\mathrm{n}=80\end{array}$ & $\begin{array}{l}\text { Grupo de tratamento } \\
\text { (fisioterapia } \\
\text { convencional e } \\
\text { treinamento em esteira) } \\
(\mathrm{n}=39)\end{array}$ & $\begin{array}{l}\text { Fisioterapia } \\
\text { convencional } \\
\text { isolada }(n=40)\end{array}$ & $\begin{array}{l}\text { Funcionalidade (HHS) } \\
\text { e força de abdutores de } \\
\text { quadril }(M R C) \text {. }\end{array}$ & $\begin{array}{l}\text { O GI obteve melhor } \\
\text { resultados para } \\
\text { funcionalidade }(p<0,0001) \\
\text { e força de abdutores do } \\
\text { quadril ( } p<0,001) \text { em } \\
\text { comparação ao GC. }\end{array}$ \\
\hline $\begin{array}{l}\text { Husby et al., } \\
2010^{41}\end{array}$ & $\begin{array}{l}\text { Ensaio Clínico } \\
\text { Randomizado } \\
\mathrm{n}=24\end{array}$ & $\begin{array}{l}\text { Grupo STG } \\
\text { (treinamento de força } \\
\text { máxima e reabilitação } \\
\text { convencional) } \\
(\mathrm{n}=12)\end{array}$ & $\begin{array}{l}\text { Grupo CRG } \\
\text { (Reabilitação } \\
\text { convencional) } \\
(n=12)\end{array}$ & $\begin{array}{l}\text { Força de extensores } \\
\text { de joelho e abdutores } \\
\text { do quadril (1RM), e } \\
\text { funcionalidade (Merle } \\
\text { d'Aubigné and Postel). }\end{array}$ & $\begin{array}{l}\text { O GI mostrou aumento } \\
\text { significativo na força do } \\
\text { quadríceps ( } p<0,002) \\
\text { e abdutores de quadril } \\
(p<0,002) \text { em comparação } \\
\text { ao GC. }\end{array}$ \\
\hline $\begin{array}{l}\text { Heiberg et } \\
\text { al., } \\
2012^{10}\end{array}$ & $\begin{array}{l}\text { Ensaio Clínico } \\
\text { Randomizado } \\
\mathrm{n}=68\end{array}$ & $\begin{array}{l}\text { Grupo treinamento } \\
\text { de habilidades de } \\
\text { caminhada }(n=35)\end{array}$ & $\begin{array}{l}\mathrm{GC} \\
(\mathrm{n}=33)\end{array}$ & $\begin{array}{l}\text { Funcionalidade (FIM e } \\
H H S \text { ), ADM de flexão, } \\
\text { extensão e abdução do } \\
\text { quadril (Goniômetro) }\end{array}$ & $\begin{array}{l}\text { O GI obteve melhora } \\
\text { significativa na ADM } \\
\text { de extensão do quadril } \\
(p=0,02) \text { e funcionalidade } \\
(p=0,001) \text { quando } \\
\text { comparado ao GC. }\end{array}$ \\
\hline $\begin{array}{l}\text { Jan et al., } \\
2004^{17}\end{array}$ & $\begin{array}{l}\text { Ensaio Clínico } \\
\mathrm{n}=53\end{array}$ & $\begin{array}{l}\text { Grupo } \\
\text { Exercício alta adesão } \\
\text { (Exercícios } \\
\text { convencionais em casa - } \\
\text { grupo com alta adesão) } \\
\text { (n=13) } \\
\text { Grupo } \\
\text { Exercício baixa adesão } \\
\text { (Exercícios } \\
\text { convencionais em } \\
\text { casa - grupo com baixa } \\
\text { adesão) } \\
\text { (n=13) }\end{array}$ & $\begin{array}{l}\mathrm{GC} \\
(\mathrm{n}=27)\end{array}$ & $\begin{array}{l}\text { Força dos flexores } \\
\text { e extensores do } \\
\text { quadril (dinamômetro } \\
\text { isocinético) e } \\
\text { funcionalidade }(H H S) \text {. }\end{array}$ & $\begin{array}{l}\text { O grupo de exercício de } \\
\text { alta adesão apresentou } \\
\text { melhora }(p<0,05) \mathrm{da} \\
\text { força dos músculos do } \\
\text { quadril bilateralmente e da } \\
\text { funcionalidade. }\end{array}$ \\
\hline $\begin{array}{l}\text { Trudelle- } \\
\text { Jackson e } \\
\text { Smith, } 2004^{11}\end{array}$ & $\begin{array}{l}\text { Ensaio Clínico } \\
\text { Randomizado } \\
\mathrm{n}=34\end{array}$ & $\begin{array}{l}\text { Grupo experimental } \\
\text { (exercícios de força e } \\
\text { estabilidade postural) } \\
(\mathrm{n}=18)\end{array}$ & $\begin{array}{l}\text { Exercícios } \\
\text { isométricos e } \\
\text { de amplitude de } \\
\text { movimento } \\
(\mathrm{n}=16)\end{array}$ & $\begin{array}{l}\text { Funcionalidade (12-Item } \\
\text { Hip Questionnaire) e } \\
\text { força muscular dos } \\
\text { extensores e flexores } \\
\text { do joelho, extensores, } \\
\text { flexores e abdutores } \\
\text { do quadril (plataforma } \\
\text { de força com o software } \\
\text { BEP). }\end{array}$ & $\begin{array}{l}\text { Houve melhora significativa } \\
\text { da funcionalidade }(p<0,01) \text {, } \\
\text { força de flexão, extensão } \\
\text { e abdução }(p<0,05) \text { do } \\
\text { quadril e de extensores de } \\
\text { joelho noGI. Não houve } \\
\text { diferenças significativas } \\
\text { para o GC }\end{array}$ \\
\hline $\begin{array}{l}\text { Stockton e } \\
\text { Mengersen, } \\
2009^{6}\end{array}$ & $\begin{array}{l}\text { Ensaio Clínico } \\
\mathrm{n}=57\end{array}$ & $\begin{array}{l}\text { Grupo tratamento } \\
\text { (fisioterapia } \\
\text { convencional e } \\
\text { exercícios funcionais } \\
\text { duas vezes ao dia) } \\
(\mathrm{n}=30)\end{array}$ & $\begin{array}{l}\text { Uma vez ao } \\
\text { dia fisioterapia } \\
\text { convencional } \\
(\mathrm{n}=27)\end{array}$ & $\begin{array}{l}\text { Funcionalidade (Iowa } \\
\text { Level of Assistance hip } \\
\text { score Oxford). }\end{array}$ & $\begin{array}{l}\text { Não houve diferença entre } \\
\text { os grupos. }\end{array}$ \\
\hline
\end{tabular}


Continuação da Tabela 1

\begin{tabular}{|c|c|c|c|c|c|}
\hline Autor, Ano & $\begin{array}{l}\text { Desenho do } \\
\text { estudo e sujeitos }\end{array}$ & $\begin{array}{l}\text { Grupo Intervenção } \\
\text { (GI) }\end{array}$ & $\begin{array}{l}\text { Grupo Controle } \\
\text { (GC) }\end{array}$ & Variáveis estudadas & Resultados \\
\hline $\begin{array}{l}\text { Sashika et } \\
\text { al.,1996 }\end{array}$ & $\begin{array}{l}\text { Ensaio } \\
\text { Clínico não } \\
\text { randomizado } \\
\mathrm{n}=23\end{array}$ & $\begin{array}{l}\text { Grupo A } \\
\text { (ADM e exercícios } \\
\text { isométricos) } \\
(\mathrm{n}=8) \\
\text { Grupo B } \\
\text { (ADM, Exercícios } \\
\text { isométricos e } \\
\text { excêntricos) } \\
(\mathrm{n}=8)\end{array}$ & $(n=7)$ & $\begin{array}{l}\text { Força de extensores } \\
\text { de joelho e flexores } \\
\text { de quadril }(M M T) \text {, } \\
\text { força isométrica } \\
\text { máxima de abdução do } \\
\text { quadril (dinamômetro } \\
\text { isocinético) e ADM } \\
\text { (JOA hip score). }\end{array}$ & $\begin{array}{l}\text { O torque isométrico } \\
\text { máximo de abdução do } \\
\text { quadril aumentou nos três } \\
\text { grupos (Grupo A: } p<0,01 ; \\
\text { Grupo B: } p<0,01 \text {; Controle: } \\
p<0,05 \text { ). } \\
\text { Sem diferença entre os } \\
\text { grupos. }\end{array}$ \\
\hline $\begin{array}{l}\text { Giaquinto et } \\
\text { al., } 2010^{16}\end{array}$ & $\begin{array}{l}\text { Estudo } \\
\text { de coorte } \\
\text { intervencional } \\
\text { prospectivo } \\
\mathrm{n}=70\end{array}$ & $\begin{array}{l}\text { Grupo HTG } \\
\text { (Grupo hidroterapia) } \\
(\mathrm{n}=31)\end{array}$ & $\begin{array}{l}\text { Grupo NHTG } \\
\text { (Exercícios } \\
\text { convencionais } \\
\text { - sem } \\
\text { hidroterapia) } \\
(\mathrm{n}=33)\end{array}$ & $\begin{array}{l}\text { Funcionalidade } \\
(W O M A C) \text {. }\end{array}$ & $\begin{array}{l}\text { HTG teve melhores } \\
\text { resultados para a } \\
\text { funcionalidade } \\
(p<0,01) \text { em comparação ao } \\
\text { grupo NHTG. }\end{array}$ \\
\hline $\begin{array}{l}\text { Galea et al., } \\
2008^{18}\end{array}$ & $\begin{array}{l}\text { Ensaio Clínico } \\
\mathrm{n}=23\end{array}$ & $\begin{array}{l}\text { Exercício não } \\
\text { supervisionado }(\mathrm{n}=12) \\
\text { Exercício } \\
\text { supervisionado }(\mathrm{n}=11)\end{array}$ & - & $\begin{array}{l}\text { Funcionalidade } \\
(W O M A C) \text {. }\end{array}$ & $\begin{array}{l}\text { Não houve diferença } \\
\text { significativa entre os grupos }\end{array}$ \\
\hline $\begin{array}{l}\text { Tsukagoshi } \\
\text { et al., } 2014^{30}\end{array}$ & $\begin{array}{l}\text { Ensaio Clínico } \\
\text { Randomizado } \\
\mathrm{n}=65\end{array}$ & $\begin{array}{l}\text { Grupo WB } \\
\text { (Suporte de peso) } \\
(\mathrm{n}=22) \\
\text { Grupo NWB } \\
(\text { Sem suporte de peso) } \\
(\mathrm{n}=21)\end{array}$ & $(\mathrm{n}=22)$ & $\begin{array}{l}\text { Funcionalidade }(H H S) \\
\text { e força muscular } \\
\text { isométrica de extensores } \\
\text { de joelho e abdutores, } \\
\text { flexores e extensores de } \\
\text { quadril (dinamômetro } \\
\text { manual). }\end{array}$ & $\begin{array}{l}\text { Houve melhora significativa } \\
\text { para a funcionalidade } \\
(p<0,01) \text { para o grupo WB } \\
\text { em comparação com o GC. } \\
\text { Não houve diferenças } \\
\text { significativas entre o WB e } \\
\text { NWB }\end{array}$ \\
\hline $\begin{array}{l}\text { Barker et al., } \\
2013^{31}\end{array}$ & $\begin{array}{l}\text { Ensaio Clínico } \\
\text { Randomizado } \\
\mathrm{n}=80\end{array}$ & $\begin{array}{l}\text { Grupo tratamento } \\
\text { (Protocolo sob medida) } \\
(\mathrm{n}=40)\end{array}$ & $\begin{array}{l}\text { Protocolo } \\
\text { Tradicional } \\
(\mathrm{n}=40)\end{array}$ & $\begin{array}{l}\text { ADM de flexão, } \\
\text { extensão e abdução de } \\
\text { quadril (Goniômetro), } \\
\text { Força muscular de } \\
\text { flexores, extensores e } \\
\text { abdutores de quadril } \\
\text { (dinamômetro de } \\
\text { mão) e funcionalidade } \\
\text { (UCLA, OHS, HOOS, } \\
\text { EuroQol) }\end{array}$ & $\begin{array}{l}\text { Houve melhora significativa } \\
\text { da funcionalidade }(p<0,011) \\
\text { para o grupo tratamento } \\
\text { em comparação com o GC. } \\
\text { Houve também melhora } \\
\text { significativa da ADM de } \\
\text { flexão, extensão }(p<0,0005) \\
\text { e abdução ( } p<0,004) \\
\text { do quadril para o GI } \\
\text { comparado com o GC }\end{array}$ \\
\hline $\begin{array}{l}\text { Rahmann et } \\
\text { al.,2009 } 209^{35}\end{array}$ & $\begin{array}{l}\text { Ensaio Clínico } \\
\text { Randomizado } \\
\mathrm{n}=54\end{array}$ & $\begin{array}{l}\text { Programa de exercícios } \\
\text { aquáticos } \\
\text { (metrônomo de ritmo } \\
\text { acelerado } 80-88 \mathrm{bpm} \text { ) } \\
(\mathrm{n}=18) \\
\text { Programa de exercícios } \\
\text { aquáticos } \\
\text { (metrônomo de ritmo } \\
\text { lento } 50-58 \mathrm{bpm} \text { ) ( } \mathrm{n}=19)\end{array}$ & $\begin{array}{l}\text { Programa de } \\
\text { exercícios da } \\
\text { convencionais } \\
\text { durante a } \\
\text { internação } \\
\text { hospitarlar } \\
(\mathrm{n}=17)\end{array}$ & $\begin{array}{l}\text { Força dos abdutores do } \\
\text { quadril e extensores do } \\
\text { joelho (dinamômetro da } \\
\text { mão), ADM de flexão } \\
\text { do joelho (Goniômetro) } \\
\text { e funcionalidade } \\
\text { (WOMAC). }\end{array}$ & $\begin{array}{l}\text { Houve diferença } \\
\text { significativa para a força } \\
\text { de abdutores do quadril } \\
\text { para o grupo que realizou } \\
\text { exercícios aquáticos com } \\
\text { metrônomo de ritmo } \\
\text { acelerado em comparação } \\
\text { com o grupo de exercícios } \\
\text { convencionais } \\
\text { ( } p=0,001) \text { e em comparação } \\
\text { com o grupo de exercício } \\
\text { aquático de ritmo lento } \\
(p=0,011) .\end{array}$ \\
\hline
\end{tabular}


Continuação da Tabela 1

\begin{tabular}{|c|c|c|c|c|c|}
\hline Autor, Ano & $\begin{array}{l}\text { Desenho do } \\
\text { estudo e sujeitos }\end{array}$ & $\begin{array}{l}\text { Grupo Intervenção } \\
(\mathrm{GI})\end{array}$ & $\begin{array}{l}\text { Grupo Controle } \\
\text { (GC) }\end{array}$ & Variáveis estudadas & Resultados \\
\hline $\begin{array}{l}\text { Unlu et al., } \\
2007^{42}\end{array}$ & $\begin{array}{l}\text { Ensaio Clínico } \\
\text { Randomizado } \\
\mathrm{n}=26\end{array}$ & $\begin{array}{l}\text { Programa de exercícios } \\
\text { em casa } \\
\text { (Exercício } \\
\text { convencional) }(\mathrm{n}=9) \\
\text { Exercícios } \\
\text { supervisionados no } \\
\text { hospital } \\
\text { (Exercício } \\
\text { convencional) }(\mathrm{n}=8)\end{array}$ & $(\mathrm{n}=9)$ & $\begin{array}{l}\text { Força de abdução de } \\
\text { quadril (dinamômetro } \\
\text { isocinético). }\end{array}$ & $\begin{array}{l}\text { O torque isométrico } \\
\text { máximo de abdução } \\
\text { do quadril melhorou } \\
\text { significativamente nos } \\
\text { grupos supervisionado } \\
\text { ( } p=0,012) \text { e terapia } \\
\text { domiciliar ( } p=0,018) . \text { O } \\
\text { grupo supervisionado } \\
\text { apresentou melhores } \\
\text { resultados para o torque de } \\
\text { abdução }(p=0,006) .\end{array}$ \\
\hline $\begin{array}{l}\text { Jogi et al., } \\
2015^{33}\end{array}$ & $\begin{array}{l}\text { Ensaio Clínico } \\
\mathrm{n}=63\end{array}$ & $\begin{array}{l}\text { Grupo Exercício } \\
\text { convencional }+ \\
\text { Equilíbrio } \\
(\mathrm{n}=13)\end{array}$ & $\begin{array}{l}\text { Grupo } \\
\text { Exercício } \\
\text { convencional } \\
\text { isolado } \\
(\mathrm{n}=17)\end{array}$ & $\begin{array}{l}\text { Funcionalidade } \\
(W O M A C) \text {. }\end{array}$ & $\begin{array}{l}\text { Houve melhora significativa } \\
\text { da funcionalidade nos dois } \\
\text { grupos ( } p<0,01) \text {. Não houve } \\
\text { diferença entre os grupos. }\end{array}$ \\
\hline $\begin{array}{l}\text { Husby et al., } \\
2009^{10}\end{array}$ & $\begin{array}{l}\text { Ensaio Clínico } \\
\text { Randomizado } \\
\mathrm{n}=24\end{array}$ & $\begin{array}{l}\text { Exercícios } \\
\text { convencionais e de } \\
\text { força máxima (STG) } \\
(\mathrm{n}=12)\end{array}$ & $\begin{array}{l}\text { Exercícios } \\
\text { convencionais } \\
\text { isolados (CRG) } \\
(\mathrm{n}=12)\end{array}$ & $\begin{array}{l}\text { 1RM - leg press; } \\
\text { Força dos abdutores } \\
\text { do quadril (1RM) e } \\
\text { funcionalidade (Merle } \\
\text { d'Aubigné and Postel) }\end{array}$ & $\begin{array}{l}\text { Houve melhora significativa } \\
\text { no teste de 1RM -Leg } \\
\text { press para membro não } \\
\text { operado para o grupo STG } \\
(p=0,044) \text { em comparação } \\
\text { com o CRG. } \\
\text { Após } 6 \text { a } 12 \text { meses, a força } \\
\text { de abdução do quadril no } \\
\text { membro operado melhorou } \\
\text { no CRG }(p=0,031) .\end{array}$ \\
\hline $\begin{array}{l}\text { Temfemo et } \\
\text { al., } 2008^{22}\end{array}$ & $\begin{array}{l}\text { Ensaio Clínico } \\
\mathrm{n}=81\end{array}$ & $\begin{array}{l}\text { Reabilitação } \\
\text { convencional } \\
\text { e exercícios isométricos } \\
\text { com feedback } \\
\text { eletromiográfico } \\
(\mathrm{n}=40)\end{array}$ & $\begin{array}{l}\text { Reabilitação } \\
\text { convencional } \\
\text { isolada }(n=41)\end{array}$ & $\begin{array}{l}\text { Força isométrica } \\
\text { voluntária máxima do } \\
\text { quadril (dinamômetro } \\
\text { isocinético). }\end{array}$ & $\begin{array}{l}\text { A adição de exercícios com } \\
\text { feedback eletromiográfico } \\
\text { proporciona aumento da } \\
\text { força do glúteo médio no } \\
\text { membro operado sete dias } \\
\text { após a cirurgia }(p<0,001) \text {. }\end{array}$ \\
\hline $\begin{array}{l}\text { Umpierres et } \\
\text { al., } 2014^{7}\end{array}$ & $\begin{array}{l}\text { Ensaio Clínico } \\
\text { Randomizado } \\
\text { n=106 }\end{array}$ & $\begin{array}{l}\text { Grupo exercício e } \\
\text { orientações (THAPCP) } \\
(n=54)\end{array}$ & $\begin{array}{l}\text { Grupo } \\
\text { orientações } \\
\text { isolada (THAP) } \\
(\mathrm{n}=52)\end{array}$ & $\begin{array}{l}\text { Funcionalidade (Merle } \\
\text { d'Aubigné and Postel), } \\
\text { ADM de flexão, } \\
\text { extensão, adução, } \\
\text { abdução e rotação } \\
\text { medial e lateral do } \\
\text { quadril (Goniômetro), } \\
\text { força muscular de } \\
\text { flexores e extensores de } \\
\text { joelho, flexão, extensão, } \\
\text { adução, abdução, } \\
\text { rotação interna e } \\
\text { externa do quadril } \\
\text { (Teste de Kendall). }\end{array}$ & $\begin{array}{l}\text { O grupo THAPCP obteve } \\
\text { melhores resultados para } \\
\text { força de flexão, extensão } \\
(p<0,001) \text {, adução } \\
(p=0,003) \text {, abdução } \\
(p=0,002) \text {, rotação interna e } \\
\text { rotação e externa de quadril } \\
\text { e funcionalidade } \\
(p<0,001)\end{array}$ \\
\hline
\end{tabular}


Continuação da Tabela 1

\begin{tabular}{|c|c|c|c|c|c|}
\hline Autor, Ano & $\begin{array}{l}\text { Desenho do } \\
\text { estudo e sujeitos }\end{array}$ & $\begin{array}{l}\text { Grupo Intervenção } \\
\text { (GI) }\end{array}$ & $\begin{array}{l}\text { Grupo Controle } \\
\text { (GC) }\end{array}$ & Variáveis estudadas & Resultados \\
\hline $\begin{array}{l}\text { Wójcik et al., } \\
2012^{21}\end{array}$ & Ensaio Clínico & $\begin{array}{l}\text { Grupo experimental } \\
\text { (exercícios terapêuticos } \\
\text { convencionais e } \\
\text { liberação fascial) }(\mathrm{n}=25)\end{array}$ & $\begin{array}{l}\text { Exercícios } \\
\text { terapêuticos } \\
\text { convencionais } \\
(\mathrm{n}=10)\end{array}$ & $\begin{array}{l}\text { ADM de flexão, } \\
\text { extensão, abdução, } \\
\text { adução, rotação interna } \\
\text { e externa do quadril } \\
\text { (Goniômetro). }\end{array}$ & $\begin{array}{l}\text { O GI apresentou um } \\
\text { aumento significativo da } \\
\text { ADM de abdução }(p=0,04) \text {, } \\
\text { adução ( } p=0,01) \text { e rotação } \\
\text { interna ( } p=0,03) \text { de quadril } \\
\text { em comparação ao GC. }\end{array}$ \\
\hline $\begin{array}{l}\text { Liebs et al., } \\
2010^{25}\end{array}$ & $\begin{array}{l}\text { Ensaio Clínico } \\
\text { Randomizado } \\
\mathrm{n}=203\end{array}$ & $\begin{array}{l}\text { Exercício com } \\
\text { cicloergômetro } \\
\text { e tratamento } \\
\text { convencional }(n=99)\end{array}$ & $\begin{array}{l}\text { Tratamento } \\
\text { convencional } \\
\text { isolado } \\
(n=104)\end{array}$ & $\begin{array}{l}\text { Functionality } \\
(W O M A C) .\end{array}$ & $\begin{array}{l}\text { O GI obteve melhora } \\
\text { significativa para a } \\
\text { funcionalidade }(p=0,046) \\
\text { em comparação ao GC. }\end{array}$ \\
\hline $\begin{array}{l}\text { Suetta et al., } \\
2004^{20}\end{array}$ & $\begin{array}{l}\text { Ensaio Clínico } \\
\text { Randomizado } \\
\mathrm{n}=36\end{array}$ & $\begin{array}{l}\text { Estimulação elétrica } \\
(\mathrm{n}=11) \\
\text { Treinamento resistido } \\
(\mathrm{n}=13)\end{array}$ & $\begin{array}{l}\text { Exercícios em } \\
\text { casa }(n=12)\end{array}$ & $\begin{array}{l}\text { Força muscular } \\
\text { isocinética de } \\
\text { extensores de joelho } \\
\text { (dinamômetro } \\
\text { isocinético) }\end{array}$ & $\begin{array}{l}\text { O grupo treinamento } \\
\text { resistido obteve melhores } \\
\text { resultados para a força } \\
\text { muscular extensora } \\
(p<0,05) .\end{array}$ \\
\hline $\begin{array}{l}\text { Suetta et al., } \\
2008^{38}\end{array}$ & $\begin{array}{l}\text { Ensaio Clínico } \\
\text { Randomizado } \\
\mathrm{n}=36\end{array}$ & $\begin{array}{l}\text { Treinamento resistido } \\
(\mathrm{n}=13) \\
\text { Estimulação elétrica } \\
(\mathrm{n}=12)\end{array}$ & $\begin{array}{l}\text { Reabilitação } \\
\text { convencional } \\
(\mathrm{n}=11)\end{array}$ & $\begin{array}{l}\text { Força muscular } \\
\text { isocinética de } \\
\text { extensores de joelho } \\
\text { (dinamômetro } \\
\text { isocinético) }\end{array}$ & $\begin{array}{l}\text { O grupo treinamento } \\
\text { resistido obteve melhores } \\
\text { resultados para a força } \\
\text { muscular extensora } \\
(p<0,05) \text {. }\end{array}$ \\
\hline $\begin{array}{l}\text { Suetta et al., } \\
2004^{37}\end{array}$ & $\begin{array}{l}\text { Ensaio Clínico } \\
\mathrm{n}=30\end{array}$ & $\begin{array}{l}\text { Reabilitação } \\
\text { convencional e } \\
\text { treinamento resistido } \\
(\mathrm{n}=11) \\
\text { Reabilitação } \\
\text { convencional e } \\
\text { estimulação elétrica } \\
\text { neuromuscular }(\mathrm{n}=10)\end{array}$ & $\begin{array}{l}\text { Reabilitação } \\
\text { convencional } \\
(\mathrm{n}=9)\end{array}$ & $\begin{array}{l}\text { Força muscular } \\
\text { isocinética de } \\
\text { extensores de joelho } \\
\text { (dinamômetro } \\
\text { isocinético) }\end{array}$ & $\begin{array}{l}\text { O grupo treinamento } \\
\text { resistido obteve melhores } \\
\text { resultados para a força } \\
\text { extensora }(p<0,01) \text {. }\end{array}$ \\
\hline $\begin{array}{l}\text { Mikkelsen et } \\
\text { al., } 2014^{44}\end{array}$ & $\begin{array}{l}\text { Ensaio Clínico } \\
\text { Randomizado } \\
\mathrm{n}=62\end{array}$ & $\begin{array}{l}\text { Grupo intervenção } \\
\text { (exercício domiciliar } \\
\text { e treinamento de } \\
\text { resistência progressiva) } \\
(\mathrm{n}=32)\end{array}$ & $\begin{array}{l}\text { Exercício em } \\
\text { casa) } \\
(\mathrm{n}=30)\end{array}$ & $\begin{array}{l}\text { Força dos abdutores } \\
\text { e flexores do quadril } \\
\text { (dinamômetro manual) } \\
\text { e funcionalidade } \\
\text { (HOOS). }\end{array}$ & $\begin{array}{l}\text { Não houve diferença entre } \\
\text { os grupos para todas as } \\
\text { variáveis analisadas. }\end{array}$ \\
\hline $\begin{array}{l}\text { Nankaku et } \\
\text { al., } 2016^{43}\end{array}$ & $\begin{array}{l}\text { Ensaio Clínico } \\
\mathrm{n}=28\end{array}$ & $\begin{array}{l}\text { Grupo exercício } \\
\text { (Reabilitação } \\
\text { convencional e exercício } \\
\text { de rotadores externos) } \\
(\mathrm{n}=14)\end{array}$ & $\begin{array}{l}\text { Reabilitação } \\
\text { convencional } \\
(n=14)\end{array}$ & $\begin{array}{l}\text { ADM de flexão e } \\
\text { abdução do quadril } \\
\text { (Goniômetro), força de } \\
\text { extensores de joelho e } \\
\text { de rotadores externos } \\
\text { e abdutores do quadril } \\
\text { (dinamômetro de mão). }\end{array}$ & $\begin{array}{l}\text { A força de abdutores do } \\
\text { quadril }(p<0,05) \text { melhorou } \\
\text { significativamente no GI } \\
\text { após a intervenção. }\end{array}$ \\
\hline $\begin{array}{l}\text { Monticone et } \\
\text { al., } 2014^{24}\end{array}$ & $\begin{array}{l}\text { Ensaio Clínico } \\
\text { Randomizado } \\
\mathrm{n}=100\end{array}$ & $\begin{array}{l}\text { Grupo experimental } \\
\text { (exercícios orientados } \\
\text { e abandonar qualquer } \\
\text { suporte de caminhada) } \\
(\mathrm{n}=50)\end{array}$ & $\begin{array}{l}\text { Exercícios de } \\
\text { cadeia aberta, } \\
\text { suporte parcial } \\
\text { de peso e uso } \\
\text { de suporte de } \\
\text { caminhada. } \\
(\mathrm{n}=50)\end{array}$ & $\begin{array}{l}\text { Funcionalidade } \\
(W O M A C)\end{array}$ & $\begin{array}{l}\text { Houve diferença } \\
\text { significativa para } \\
\text { funcionalidade }(p<0,001) \\
\text { no GI. }\end{array}$ \\
\hline
\end{tabular}


Continuação da Tabela 1

\begin{tabular}{|c|c|c|c|c|c|}
\hline Autor, Ano & $\begin{array}{l}\text { Desenho do } \\
\text { estudo e sujeitos }\end{array}$ & $\begin{array}{l}\text { Grupo Intervenção } \\
\text { (GI) }\end{array}$ & $\begin{array}{l}\text { Grupo Controle } \\
\text { (GC) }\end{array}$ & Variáveis estudadas & Resultados \\
\hline $\begin{array}{l}\text { Pohl et al., } \\
2015^{32}\end{array}$ & $\begin{array}{l}\text { Ensaio Clínico } \\
\mathrm{n}=58\end{array}$ & 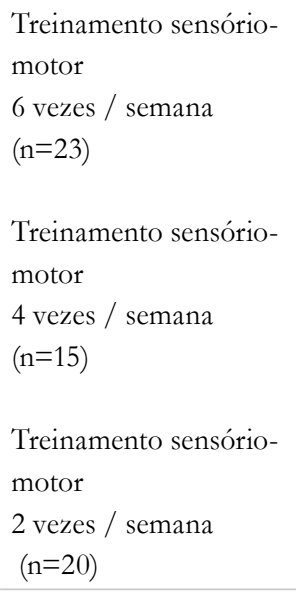 & - & $\begin{array}{l}\text { Funcionalidade } \\
\text { (Lequesne). }\end{array}$ & $\begin{array}{l}\text { Houve melhora significativa } \\
\text { na funcionalidade ao longo } \\
\text { do tempo ( } p<0,001) \text {, mas } \\
\text { não diferiu entre os grupos. }\end{array}$ \\
\hline $\begin{array}{l}\text { Smith et al., } \\
2008^{29}\end{array}$ & $\begin{array}{l}\text { Ensaio Clínico } \\
\text { Randomizado } \\
\mathrm{n}=60\end{array}$ & $\begin{array}{l}\text { Reeducação da marcha } \\
\text { e exercício na cama } \\
(\mathrm{n}=30)\end{array}$ & $\begin{array}{l}\text { Reeducação da } \\
\text { marcha (isolado) } \\
(\mathrm{n}=30)\end{array}$ & Funcionalidade (ILOA). & $\begin{array}{l}\text { Não houve diferença entre } \\
\text { os grupos para todas as } \\
\text { medidas de resultado. }\end{array}$ \\
\hline $\begin{array}{l}\text { Matheis } \\
\text { e Stöggl, } \\
2018^{50}\end{array}$ & $\begin{array}{l}\text { Ensaio Clínico } \\
\mathrm{n}=39\end{array}$ & $\begin{array}{l}\text { Grupo intervenção } \\
\text { (mobilização e } \\
\text { treinamento de força) } \\
(\mathrm{n}=20)\end{array}$ & $(n=19)$ & $\begin{array}{l}\text { ADM de flexão, } \\
\text { abdução e extensão do } \\
\text { quadril (Goniômetro), } \\
\text { funcionalidade (Merle } \\
\text { d'Aubigné, HHS e } \\
W O M A C \text { ). }\end{array}$ & $\begin{array}{l}\text { O GI apresentou melhora } \\
\text { significativa para ADM de } \\
\text { flexão }(p<0,01) \text {, extensão } \\
(p<0,001) \text { e abdução de } \\
\text { quadril }(p<0,01) \text {. }\end{array}$ \\
\hline $\begin{array}{l}\text { Winther et } \\
\text { al., } 2018^{39}\end{array}$ & $\begin{array}{l}\text { Ensaio Clínico } \\
\text { Randomizado } \\
\mathrm{n}=60\end{array}$ & $\begin{array}{l}\text { Fisioterapia } \\
\text { convencional } \\
(n=29)\end{array}$ & $\begin{array}{l}\text { Treinamento de } \\
\text { força maxima } \\
(\mathrm{n}=31)\end{array}$ & $\begin{array}{l}\text { Funcionalidade (HOOS } \\
\text { eHHS) e força dos } \\
\text { abdutores (aparelho de } \\
\text { tração) }\end{array}$ & $\begin{array}{l}\text { O GI apresentou melhora } \\
\text { significativa da força dos } \\
\text { abdutores }(p \leq 0,002)\end{array}$ \\
\hline $\begin{array}{l}\text { Monaghan et } \\
\text { al., } 2017^{26}\end{array}$ & $\begin{array}{l}\text { Ensaio Clínico } \\
\text { Randomizado } \\
\mathrm{n}=63\end{array}$ & $\begin{array}{l}\text { Grupo Exercício e } \\
\text { grupo cuidados } \\
\text { habituais }(n=32)\end{array}$ & $\begin{array}{l}\text { Cuidados } \\
\text { habituais } \\
\text { isolado) }(\mathrm{n}=31)\end{array}$ & $\begin{array}{l}\text { Funcionalidade } \\
\text { (WOMAC) } \\
\text { Funcionalidade } \\
\text { (dinamômetro). }\end{array}$ & $\begin{array}{l}\text { O GI obteve melhora } \\
\text { significativa na } \\
\text { funcionalidade }(p<0,01) \text {. }\end{array}$ \\
\hline
\end{tabular}

ATQ: Artroplastia total de quadril; ADM: Amplitude de movimento; HHS: Harris Hip Score; FIM: Functional Independence Measure; WOMAC: Western Ontario and McMaster Universities; MRC: Medical Research Council; MMT: Manual Muscular Testing; ILOA: Iowa Level of Assistance Scale; OHS: Oxford Hip Score; HOOS: Hip Disability and Osteoartbritis Outcome Score; UCLA: UCL A Activity Score; 1RM: Teste de repetição máxima.

Tabela 2. Classificação do risco de viés baseada na escala de JADAD para os estudos incluídos na revisão sistemática. Campo Grande, MS, 2019.

\begin{tabular}{|c|c|c|c|c|c|c|c|}
\hline Autor, ano & Randomizado & $\begin{array}{l}\text { Randomização } \\
\text { Adequada? }\end{array}$ & $\begin{array}{l}\text { Duplo } \\
\text { Cego }\end{array}$ & $\begin{array}{l}\text { Duplo Cego } \\
\text { Adequado? }\end{array}$ & $\begin{array}{l}\text { Perdas } \\
\text { Amostrais }\end{array}$ & Pontuação & Qualidade \\
\hline $\begin{array}{l}\text { Gremeaux et al., } \\
2008^{8}\end{array}$ & $\operatorname{Sim}$ & Sim & Não & - & Sim & 3 & Alta Qualidade \\
\hline Liebs et al., $2012^{13}$ & Sim & Sim & Não & - & Sim & 3 & Alta Qualidade \\
\hline Hesse et al., $2003^{14}$ & Sim & Sim & Não & - & Sim & 3 & Alta Qualidade \\
\hline Husby et al., $2009^{41}$ & Sim & Sim & Não & - & Sim & 3 & Alta Qualidade \\
\hline Heiberg et al., & Sim & Sim & Não & - & Sim & 3 & Alta Qualidade \\
\hline
\end{tabular}

$2012^{10}$ 
Continuação da Tabela 2

\begin{tabular}{|c|c|c|c|c|c|c|c|}
\hline Autor, ano & Randomizado & $\begin{array}{l}\text { Randomização } \\
\text { Adequada? }\end{array}$ & $\begin{array}{l}\text { Duplo } \\
\text { Cego }\end{array}$ & $\begin{array}{l}\text { Duplo Cego } \\
\text { Adequado? }\end{array}$ & $\begin{array}{l}\text { Perdas } \\
\text { Amostrais }\end{array}$ & Pontuação & Qualidade \\
\hline Jan et al., $2004^{17}$ & $\operatorname{Sim}$ & Não & Não & - & Sim & 1 & Baixa Qualidade \\
\hline Trudelle Jackson e & & & & & & & Alta Qualidade \\
\hline Smith, $2004^{11}$ & $\operatorname{Sim}$ & $\operatorname{Sim}$ & Não & - & Sim & 3 & \\
\hline $\begin{array}{l}\text { Stockton e } \\
\text { Mengersen } 2009^{6}\end{array}$ & Sim & Não & Não & - & $\operatorname{Sim}$ & 1 & Baixa Qualidade \\
\hline Sashika et al.,19969 & Não & - & Não & - & $\operatorname{Sim}$ & 1 & Baixa Qualidade \\
\hline $\begin{array}{l}\text { Giaquinto et al., } \\
2010^{16}\end{array}$ & $\operatorname{Sim}$ & Não & Não & - & $\operatorname{Sim}$ & 1 & Baixa Qualidade \\
\hline Galea et al., $2008^{18}$ & $\operatorname{Sim}$ & Não & Não & - & $\operatorname{Sim}$ & 1 & Baixa Qualidade \\
\hline $\begin{array}{l}\text { Tsukagoshi et al., } \\
2014^{30}\end{array}$ & Sim & $\operatorname{Sim}$ & Não & - & $\operatorname{Sim}$ & 3 & Alta Qualidade \\
\hline Barker et al., $2013^{31}$ & $\operatorname{Sim}$ & $\operatorname{Sim}$ & Não & - & $\operatorname{Sim}$ & 3 & Alta Qualidade \\
\hline $\begin{array}{l}\text { Rahmann et } \\
\text { al.,2009 }\end{array}$ & Sim & $\operatorname{Sim}$ & Não & - & $\operatorname{Sim}$ & 3 & Alta Qualidade \\
\hline Unlu et al., $2007^{42}$ & $\operatorname{Sim}$ & $\operatorname{Sim}$ & Não & - & $\operatorname{Sim}$ & 3 & Alta Qualidade \\
\hline Jogi et al., $2015^{33}$ & $\operatorname{Sim}$ & Não & Não & - & $\operatorname{Sim}$ & 1 & Baixa Qualidade \\
\hline Husby et al., $2010^{10}$ & $\operatorname{Sim}$ & $\operatorname{Sim}$ & Não & - & $\operatorname{Sim}$ & 3 & Alta Qualidade \\
\hline $\begin{array}{l}\text { Temfemo et al., } \\
2008^{22}\end{array}$ & Não & - & Não & - & Não & 0 & Baixa Qualidade \\
\hline $\begin{array}{l}\text { Umpierres et al., } \\
2014^{7}\end{array}$ & Sim & $\operatorname{Sim}$ & Sim & Sim & Não & 4 & Alta Qualidade \\
\hline Wójcik et al., $2012^{21}$ & Não & - & Não & - & Não & 0 & Baixa Qualidade \\
\hline Liebs et al., $2010^{25}$ & $\operatorname{Sim}$ & $\operatorname{Sim}$ & Não & - & $\operatorname{Sim}$ & 3 & Alta Qualidade \\
\hline Suetta et al., $2004^{20}$ & $\operatorname{Sim}$ & $\operatorname{Sim}$ & $\operatorname{Sim}$ & $\operatorname{Sim}$ & $\operatorname{Sim}$ & 5 & Alta Qualidade \\
\hline Suetta et al., $2008^{38}$ & $\operatorname{Sim}$ & $\operatorname{Sim}$ & Não & - & $\operatorname{Sim}$ & 3 & Alta Qualidade \\
\hline Suetta et al., $2004^{37}$ & $\operatorname{Sim}$ & Não & Não & - & $\operatorname{Sim}$ & 1 & Baixa Qualidade \\
\hline $\begin{array}{l}\text { Mikkelsen et al., } \\
2014^{44}\end{array}$ & Sim & $\operatorname{Sim}$ & Não & - & $\operatorname{Sim}$ & 3 & Alta Qualidade \\
\hline $\begin{array}{l}\text { Nankaku et al., } \\
2016^{43}\end{array}$ & $\operatorname{Sim}$ & Não & Não & - & $\operatorname{Sim}$ & 1 & Baixa Qualidade \\
\hline $\begin{array}{l}\text { Monticone et al., } \\
2014^{24}\end{array}$ & $\operatorname{Sim}$ & $\operatorname{Sim}$ & Não & - & $\operatorname{Sim}$ & 3 & Alta Qualidade \\
\hline Pohl et al., $2015^{32}$ & $\operatorname{Sim}$ & Não & Não & - & $\operatorname{Sim}$ & 1 & Baixa Qualidade \\
\hline Smith et al., $2008^{29}$ & $\operatorname{Sim}$ & $\operatorname{Sim}$ & Não & - & $\operatorname{Sim}$ & 3 & Alta Qualidade \\
\hline $\begin{array}{l}\text { Matheis e Stöggl, } \\
2018^{50}\end{array}$ & $\operatorname{Sim}$ & Não & Não & & $\operatorname{Sim}$ & 1 & Baixa Qualidade \\
\hline $\begin{array}{l}\text { Winther et al., } \\
2018^{39}\end{array}$ & $\operatorname{Sim}$ & $\operatorname{Sim}$ & No & & $\operatorname{Sim}$ & 3 & Alta Qualidade \\
\hline $\begin{array}{l}\text { Monaghan et al., } \\
2017^{26}\end{array}$ & $\operatorname{Sim}$ & $\operatorname{Sim}$ & No & & $\operatorname{Sim}$ & 3 & Alta Qualidade \\
\hline
\end{tabular}




\section{Desfechos Analisados}

Considerando-se os desfechos em comum, 23 artigos avaliaram a funcionalidade, 21 artigos avaliaram a força muscular e oito analisaram a amplitude de movimento.

\section{DISCUSSÃO}

O presente estudo foi proposto para descrever os efeitos da fisioterapia sobre estimadores de funcionalidade, força e amplitude de movimento em pacientes submetidos à $\mathrm{ATQ}$, secundária à $\mathrm{OA}$. De forma geral, exercícios resistidos (dinâmicos e isométricos) de alta intensidade são mais eficazes para melhora da funcionalidade. No que diz respeito à força muscular e ADM exercícios dinâmicos com 3 a 5 séries de 8 a 12 repetições com baixa e alta intensidade promoveram ganhos mais expressivos em relação a outras modalidades terapêuticas.

\section{Funcionalidade}

Evidências revelam que pacientes submetidos à ATQ podem apresentar deficit funcionais persistentes, os quais são associados a limitações biomecânicas e alterações na cinemática da marcha, derivados do procedimento cirúrgico e/ ou quadro álgico ${ }^{23}$.

Os resultados de três estudos mostraram que exercícios funcionais baseados nos movimentos diários são capazes de restabelecer a funcionalidade de pacientes submetidos à ATQ ${ }^{10,18,24-26}$. Contudo, é importante relatar que, em geral, os exercícios funcionais utilizados pelos autores citados foram acompanhados de ciclismo e caminhada ${ }^{10,24,25}$.

Considerando-se exercícios resistidos, a literatura reporta que essa modalidade de intervenção é relevante para melhorar a funcionalidade de idosos saudáveis ${ }^{27,28}$. Neste contexto, estudos prévios revelaram que exercícios resistidos de baixa ${ }^{27} \mathrm{e}$ moderada intensidades ${ }^{28}$ são relevantes para melhorar o desempenho funcional para essa população. Em nossos achados, uma pesquisa com baixa pontuação na escala JADAD mostrou resultados positivos sobre a funcionalidade, a partir de protocolos de exercício resistido de baixa intensidade para pacientes submetidos à $\mathrm{ATQ}^{18}$. Entretanto, dois estudos considerados de alta qualidade pela escala JADAD mostraram que exercícios resistidos de baixa intensidade não repercutiram em alteração da funcionalidade ${ }^{8,15}$. Além disso, outro estudo, considerado de alta qualidade, mostrou que exercícios sem resistência externa não são efetivos para ganhos expressivos na funcionalidade ${ }^{29}$. Portanto, não está bem estabelecido qual é o potencial dos protocolos de exercício resistido, considerando-se os efeitos de diferentes cargas sobre a funcionalidade de pacientes com ATQ. Diante disso, mais estudos são necessários para elucidar qual a intensidade de exercício resistido que promove melhor recuperação funcional.

Por outro lado, exercícios dinâmicos e isométricos, aplicados como parte do tratamento fisioterapêutico de ATQ, mostraram-se eficazes em promover benefícios funcionais significativos ${ }^{7,11,14,17,29-33}$. Além disso, os resultados funcionais parecem ser ainda melhores na reabilitação após ATQ quando exercícios dinâmicos e isométricos foram acompanhados de exercícios de equilíbrio ${ }^{32,33}$. É possível observar melhora da função através de exercícios resistidos, pois ocorre aumento da força muscular, a qual é um preditor da capacidade funcional e necessária para executar a maioria das atividades da vida diária ${ }^{34}$.

Os trabalhos consultados nesta revisão mostram resultados inconclusivos sobre a eficácia de fisioterapia aquática sobre a funcionalidade de pacientes com ATQ. Husby et al. ${ }^{15}$ e Rahmann et al. ${ }^{35}$, que conduziram trabalhos classificados como de alta qualidade, compararam a influência de exercícios na piscina com exercícios em solo e verificaram que ambas as terapias promovem aumento da funcionalidade; contudo, não constataram diferenças significativas entre as duas modalidades terapêuticas. Para Stokton et al. ${ }^{6}$ e Guiaquinto et al. ${ }^{16}$, cujos trabalhos foram classificados como de baixa qualidade, a hidroterapia apresenta melhores resultados do que exercícios em solo. Ademais, segundo Liebs et al. ${ }^{25}$, a hidroterapia é efetiva para a funcionalidade, mas somente se realizada após a retirada de pontos. Portanto, as evidências científicas quanto à efetividade da hidroterapia são fracas, considerando a baixa qualidade metodológica dos estudos identificados. Mais estudos necessitam 
ser realizados para melhor esclarecer o papel da hidroterapia como recurso na ATQ.

\section{Força Muscular}

Classicamente, o quadro crônico de osteoartrite repercute em importante diminuição da força muscular. Tais deficit tornam-se ainda mais acentuados em razão dos efeitos deletérios causados pelo procedimento cirúrgico sobre a musculatura ${ }^{36}$. Portanto, o fortalecimento muscular deve compor os objetivos da reabilitação após ATQ. Os estudos incluídos nesta revisão podem contribuir para a prescrição adequada de protocolos de fortalecimento muscular após ATQ.

Em relação aos exercícios dinâmicos, é recomendada a prescrição de protocolos com duas séries de 10 repetições para que se obtenha aumento da força muscular em indivíduos destreinados ${ }^{37}$. Entre nossos achados, somente oito estudos mostraram o número de séries e repetições utilizadas em protocolos de exercícios dinâmicos ${ }^{7,9,17,20,29,37-39}$.

Para o músculo quadríceps, prescrições com 3 a 5 séries de 8 a 10 repetições foram capazes de aumentar a força em sujeitos que realizaram $\mathrm{ATQ}^{20,37,38}$. No estudo desenvolvido por Umpierres et al. ${ }^{7}$, foram aplicadas três séries com 12 repetições, evidenciando aumento da força muscular de extensores, abdutores, adutores e rotadores de quadril, flexores e extensores de joelho. No estudo realizado por Tsukagoshi et al. ${ }^{30}$, foram utilizadas três séries de 15 repetições, resultando em aumento de força para extensores do joelho, abdutores, extensores e flexores do quadril. Segundo Sashika et al. ${ }^{9}$ e Jan et al. ${ }^{17}$, duas séries de 10 repetições produzem aumento da força muscular de abdução, flexão e extensão de quadril. Esses dados mostram que protocolos com 2 a 3 séries de 8 a 12 repetições parecem seguros não somente para indivíduos hígidos, mas também para pacientes com artroplastia total de quadril.

É importante ressaltar que, além do número adequado de séries e repetições, também se faz necessário discutir a quantidade segura de carga para ganhos significativos da força muscular. As evidências revelam aumento da força com prescrições de 60 a 70\% da repetição máxima (RM) para idosos hígidos ${ }^{40}$. Esta revisão mostra resultados significativos para o ganho de força com prescrições de moderada a alta intensidade, utilizando $50 \%$, $65 \%, 70 \%, 80 \%, 85 \%$ e $90 \%$ da $\mathrm{RM}^{15,20,37-39,41}$. No entanto, esses resultados positivos também foram encontrados quando utilizados exercícios de baixa intensidade, com $10 \%, 30 \%$ e $40 \%$ da $\mathrm{RM}^{18,17,42,43}$.

Neste sentido, vale ressaltar que a prescrição de carga, seja de baixa ou alta intensidade, é muito importante, uma vez que em cinco estudos que fizeram parte desta revisão grupos de pacientes submetidos à ATQ que realizavam exercícios com carga apresentaram maior força em relação a exercícios sem carga ${ }^{20,30,37,38,44}$.

Com relação aos exercícios isométricos, sua efetividade para ganho de força em sujeitos submetidos à ATQ ainda é pouco evidenciada. Em seis estudos, o exercício isométrico foi utilizado como protocolo de tratamento ${ }^{11,22,30,31,35,42}$. Em três trabalhos, os autores encontraram ganho de força para abdutores, flexores, extensores do quadril e para extensores de joelho ${ }^{22,30,42}$. Contudo, um desses estudos recebeu baixo escore pela escala JADAD ${ }^{22}$. Três estudos considerados de alta qualidade não encontraram melhora da força no grupo que realizou isometria apenas com resistência gravitacional $1^{11,31,43}$.

A maioria dos estudos incluídos não relatou com detalhes a prescrição utilizada, impossibilitando comparações e conclusões a respeito do tipo de prescrição necessária para o ganho de força com o exercício isométrico. A literatura reporta que exercícios isométricos produzem ganhos de força quando são realizadas 6 repetições com 30 a 40 segundos de sustentação em idosos saldáveis ${ }^{45}$. Nesta revisão, foram encontrados ganhos de força para o quadríceps com prescrições de três repetições sustentadas por 20 segundos para pacientes submetidos à $\mathrm{ATQ}^{30}$. Apesar do exercício isométrico mostrar-se efetivo para induzir ganho de força, mais estudos são necessários para esclarecer o tipo de prescrição adequada para sujeitos com ATQ.

Por outro lado, o ganho de força através da hidroterapia é relatado na literatura, principalmente, 
quando associada a equipamentos para aumentar a resistência. Ademais, trata-se de uma modalidade que pode ser prescrita de forma segura, a fim de potencializar o condicionamento muscular e com diminuição do risco de lesões que podem ser causadas pelo exercício ${ }^{46}$. Neste sentido, três trabalhos estudaram os efeitos da hidroterapia sobre a força muscular em pacientes submetidos à $\mathrm{ATQ}^{15,35,41}$. De forma geral, a hidroterapia parece apresentar melhores resultados para o ganho de força quando comparada com exercícios isométricos ${ }^{44}$. Entretanto, quando associada a exercícios em solo de baixa intensidade, a hidroterapia não se mostra mais eficaz que exercícios em solo de alta intensidade ${ }^{15,41}$. Não obstante, a hidroterapia pode ser usada em estágios iniciais, quando os pacientes não suportam os exercícios praticados em terra, ou não são capazes de sustentar total ou parcialmente a massa corporal ${ }^{47}$.

Outro recurso de tratamento relatado nos estudos foi a eletroterapia do tipo bifásica com frequência de $40 \mathrm{~Hz}^{20,37,38}$ e $10 \mathrm{~Hz}^{8}$. Os resultados mostraram que o uso isolado da eletroterapia não produz aumento da força ${ }^{8,20,37,38}$. Contudo, a eletroterapia parece potencializar os efeitos provocados pelo exercício resistido sobre a força muscular do quadríceps ${ }^{8}$. Esses resultados se aproximam dos encontrados por Hauger et al. ${ }^{48}$, em que a eletroestimulação neuromuscular associada a exercícios favorece o aumento de força do quadríceps em outra condição ortopédica. Portanto, pode-se conjecturar que a eletroterapia apresenta bons resultados como coadjuvante ao exercício resistido.

Com relação à influência da presença do fisioterapeuta durante a sessão de exercícios, um estudo incluído nesta revisão sistemática concluiu que os pacientes que realizaram exercícios com supervisão apresentaram resultados mais significativos para a melhora da força de abdução, quando comparados aos grupos sem supervisão ${ }^{42}$. Considerando essa questão, Kuru et al. ${ }^{49}$ estudaram o efeito de um programa de fisioterapia supervisionada em idosos com osteoartrite do joelho. A amostra foi dividida em dois grupos que realizaram o mesmo protocolo por 6 semanas; porém, apenas um dos grupos estava sob supervisão e apresentou maior ganho de força que o grupo sem supervisão. Esses resultados sugerem que a supervisão afeta os resultados de ganho de força muscular, provavelmente porque o profissional deve controlar a velocidade de execução, os intervalos de descanso, o ajuste da carga e outras variáveis importantes para tornar o desempenho mais preciso e obter melhores resultados.

\section{Amplitude de Movimento}

Em relação à amplitude de movimento do quadril, esta revisão mostrou que exercícios resistidos com três séries, de 8 a 12 repetições, para glúteos e músculos da coxa são eficazes para aumentar a amplitude de movimento (ADM) de flexão, extensão, abdução, adução, rotação medial e lateral externa do quadri1 ${ }^{8,41}$. Quando é realizada uma prescrição com menor número de séries e repetições (duas séries de 10 repetições), não ocorre aumento significativo da $\mathrm{ADM}^{9}$.

Exercícios isométricos, quando associados a outra modalidade de exercício, também se mostraram eficazes para o aumento da ADM de flexão, extensão, abdução, rotação medial e lateral em pacientes submetidos à $\mathrm{ATQ}^{21,31}$. Nos estudos incluídos nesta revisão, os autores associaram a isometria com exercícios ativos livres, treino de marcha ${ }^{21}$ exercícios funcionais e alongamento ${ }^{31}$. Em contrapartida, quando associados com técnicas de liberação miofascial, estes resultados parecem ser ainda mais satisfatórios ${ }^{21}$.

A maioria dos estudos incluídos nesta revisão relacionou o exercício resistido com o aumento da ADM. O estudo de Fatouros et al. ${ }^{40}$ solidifica esses resultados, uma vez que encontrou aumento da flexibilidade em idosos que realizaram treinamento resistido com três séries, utilizando $40 \%$ (baixa intensidade), 60\% (moderada intensidade) e 80\% (alta intensidade) da 1RM. Os autores concluíram que o treinamento de resistência, isoladamente, melhora a flexibilidade no idoso; no entanto, melhores resultados são obtidos utilizando $60 \%$ da RM (moderada intensidade). Além disso, quando mobilização passiva e ativa é adicionada ao protocolo de exercício resistido, o ganho de amplitude articular parece evoluir mais rapidamente ${ }^{50}$. Os resultados dos estudos desta revisão também mostraram que o efeito positivo sobre o ganho de ADM é maior 
quando existe a presença do fisioterapeuta durante a realização dos exercícios ${ }^{7,10}$.

\section{CONCLUSÃO}

Em conclusão, as técnicas e protocolos usados pela fisioterapia para tratamento da ATQ são variados e têm importante eficácia clínica comprovada na literatura. Os ensaios clínicos analisados apresentaram melhora significativa nos grupos experimentais em comparação aos grupos controle em todos os desfechos avaliados (funcionalidade, força muscular e ADM). Em geral, protocolos supervisionados com a realização de exercícios ativos para os músculos periarticulares ao quadril e para extensores de joelho têm proporcionado importante prognóstico funcional. No entanto, embora a maioria dos estudos apresente alto nível de qualidade pela escala JADAD, os resultados

\section{REFERÊNCIAS}

1. Miranda GMD, Mendes ADCG, Silva ALAD. Population aging in Brazil: current and future social challenges and consequences. Rev Bras Geriatr Gerontol. 2016;19(3):507-19.

2. King M, Lipsky MS. Clinical implications of aging. Dis Mon. 2015;61(11):467-4.

3. Van der Kraan PM, Berenbaum F, Blanco FJ, de Bari C, Falaber F, Hauge E, et al. Translation of clinical problems in osteoarthritis into pathophysiological research goals. RMD Open 2016;2(1):e000224..

4. Bortoluzzi A, Furini F, Scirè CA. Osteoarthritis and its management - Epidemiology, nutritional aspects and environmental factors. Autoimmun Rev. 2018;17(11):1097-104.

5. Geeske Peeters GM, Rainbird S, Lorimer M, Dobson AJ, Mishra GD, Graves SE. Improvements in physical function and pain sustained for up to 10 years after knee or hip arthroplasty irrespective of mental health status before surgery. Acta Orthop. 2017;88(2):158-65.

6. Stockton KA, Mengersen KA. Effect of multiple physiotherapy sessions on functional outcomes in the initial postoperative period after primary total hip replacement: a randomized controlled trial. Arch Phys Med Rehabil. 2009;90(10):1652-57. devem ser analisados com cautela, uma vez que vários protocolos com diferentes combinações de terapias foram observados.

Considerando a importância da prática baseada em evidências na tomada de decisão clínica, sugere-se a realização de ensaios clínicos randomizados que especifiquem os métodos de treinamento (como tipo e velocidade da contração muscular, frequência e intensidade dos exercícios), para que no processo de reabilitação pós-cirúrgico de ATQ possam ser prescritas terapias apropriadas e seguras.

\section{AGRADECIMENTOS}

Somos gratos a Ingrid Müller Costa pela edição do inglês.

Editado por: Ana Carolina Lima Cavaletti
7. Umpierres CS, Ribeiro TA, Marchisio AE, Galvão L, Borges INK, Macedo C, et al. Rehabilitation following total hip arthroplasty evaluation over short follow-up time: Randomized clinical trial. J Phys Med Rehabil. 2014;51(10):1567-78.

8. Gremeaux V, Renault J, Pardon L, Deley G, Lepers $\mathrm{R}$, Casillas JM. Low-frequency electric muscle stimulation combined with physical therapy after total hip arthroplasty for hip osteoarthritis in elderly patients: a randomized controlled trial. Arch Phys Med Rehabil. 2008;89(12):2265-73.

9. Sashika H, Matsuba Y, Watanabe Y. Home program of physical therapy: effect on disabilities of patients with total hip arthroplasty. Arch Phys Med Rehabil. 1996;77(3):273-77.

10. Heiberg KE, Bruun-Olsen V, Ekeland A, Mengshoel AM. Effect of a walking skill training program in patients who have undergone total hip arthroplasty: Followup one year after surgery. Arthritis Care Res. 2012:64(3):415-23.

11. Trudelle-Jackson E, Smith SS. Effects of a latephase exercise program after total hip arthroplasty: a randomized controlled trial. Arch Phys Med Rehabil. 2004;85(7):1056-62. 
12. Mikkelsen LR, Mikkelsen SS, Christensen FB. Early, Intensified Home-based Exercise after Total Hip Replacement: a pilot study. Physiother Res Int. 2012;17(4):214-26.

13. Liebs TR, Herzberg W, Rüther W, Haasters J, Russlies M, Hassenpflug J. Multicenter Arthroplasty Aftercare Project. Multicenter randomized controlled trial comparing early versus late aquatic therapy after total hip or knee arthroplasty. Arch Phys Med Rehabil. 2012;93(2):192-99.

14. Hesse S, Werner C, Seibel H, Von Frankenberg S, Kappel EM, Kirker S,et al. Treadmill training with partial body-weight support after total hip arthroplasty: a randomized controlled trial. Arch Phys Med Rehabil 2003;84(12):1767-73.

15. Husby VS, Helgerud J, Bjorgen S, Husby OS, Benum P, Hoff J. Early maximal strength training is an efficient treatment for patients operated with total hip arthroplasty. Arch Phys Med Rehabil. 2009;90(10):1658-67.

16. Giaquinto S, Ciotola E, Dall'armi V, Margutti F. Hydrotherapy after total hip arthroplasty: a follow-up study. Arch Gerontol Geriatr. 2010;50(1)92-95.

17. Jan M, Hung JY, Lin JC, Wang SF, Liu TK, Tang PF. Effects of a home program on strength, walking speed, and function after total hip replacement. Arch Phys Med Rehabil. 2004;85(12):1943-51.

18. Galea MP, Levinger P, Lythgo N, Cimoli C, Weller $\mathrm{R}$, Tully E, et al. A targeted home-and centerbased exercise program for people after total hip replacement: a randomized clinical trial. Arch Phys Med Rehabil. 2008;89(8):1442-47.

19. Jadad AR, Moore RA, Carroll D, Jenkinson C, Reynolds DJ, Gavaghan DJ, et al. Assessing the quality of reports of randomized clinical trials: is blinding necessary? Control Clin Trials. 1996;17(1):1-12.

20. Suetta C, Magnusson SP, Rosted A, Aagaard P, Jakobsen AK, Larsen LH, et al. Resistance training in the early postoperative phase reduces hospitalization and leads to muscle hypertrophy in elderly hip surgery patients: a controlled, randomized study. J Am Geriatr Soc. 2004;52(12)2016-22.

21. Wójcik B, Jabłoński M, Gębala E, Drelich MA. Comparison of effectiveness of fascial relaxation and classic model of patients rehabilitation after hip joint endoprosthetics. Ortop Traumatol Rehabil. 2011;14(2):161-78.

22. Temfemo A, Doutrellot PL, Ahmaidi S. Early muscular strengthening after total hip arthroplasty: association of two models of rehabilitation. Ann Readapt Med Phys. 2008;38-45.
23. Cezarino L, Vieira W, Silva J, Silva-Filho E, Souza F, Scattone R. Gait and functionality following unilateral and bilateral hip replacement. Fisioter mov. 2019;32:1-10.

24. Monticone M, Ambrosini E, Rocca B, Lorenzon C, Ferrante S, Zatti G. Task-oriented exercises and early full weight-bearing contribute to improving disability after total hip replacement: a randomized controlled trial. Clin Rehabil. 2014;28(7):658-68.

25. Liebs TR, Herzberg W, Rüther W, Haasters J, Russlies M, Hassenpflug J. Ergometer cycling after hip or knee replacement surgery. J Bone Jt Surg. 2010;92(4):814-22.

26. Monaghan B, Cunningham P, Harrington P, Hing W, Blake C, O'Dohertya D, et al. Randomised controlled trial to evaluate a physiotherapy-led functional exercise programme after total hip replacement. Physiotherapy. 2017;103(3):283-88.

27. Sahin UK, Kirdi N, Bozoglu E, Meric A, Buyukturan G, Ozturk A, et al. Effect of low-intensity versus high-intensity resistance training on the functioning of the institutionalized frail elderly. Int J Rehabil Res. 2018;41(3):211-7.

28. Martínez-Velilla N, Casas-Herrero A, ZambomFerraresi F, de Asteasu MLS, Lucia A, Galbete A, et al. Effect of exercise intervention on functional decline in very elderly patients during acute hospitalization: a randomized clinical Trial. JAMA Intern Med. 2019;179(1):28-36.

29. Smith TO, Mann CJ, Clark A, Donell ST. Bed exercises following total hip replacement: a randomised controlled trial. Physiotherapy. 2008;94(4):286-91.

30. Tsukagoshi R, Tateuchi H, Fukumoto Y, Ibuki S, Akiyama H, So K, et al. Functional performance of female patients more than 6 months after total hip arthroplasty shows greater improvement with weight-bearing exercise than with non-weight-bearing exercise. Randomized controlled trial. Eur J Phys Rehabil Med. 2014;50(6):665-75.

31. Barker KL, Newman MA, Hughes T, Sackley C, Pandit H, Kiran A, et al. Recovery of function following hip resurfacing arthroplasty: a randomized controlled trial comparing an accelerated versus standard physiotherapy rehabilitation programme. Clin Rehabil 2013;27(9):771-84.

32. Pohl T, Brauner T, Wearing S, Stamer K, Horstmann T. Effects of sensorimotor training volume on recovery of sensorimotor function in patients following lower limb arthroplasty. BMC Musculoskelet Disorders. 2015;16(1):1-10. 
33. Jogi P, Overend TJ, Spaulding SJ, Zecevic A, Kramer JF. Effectiveness of balance exercises in the acute post-operative phase following total hip and knee arthroplasty: a randomized clinical trial. SAGE Open Med. 2015;3:1-10.

34. Stasi S, Papathanasiou G, Chronopoulos E, Galanos A, Papaioannou NA, Triantafyllopoulos IK. Association between abductor muscle strength and functional outcomes in hip-fractured patients: a cross-sectional study. J Musculoskelet Neuronal Interact. 2018;18(4):530-42.

35. Rahmann AE, Brauer SG, Nitz JC. A specific inpatient aquatic physiotherapy program improves strength after total hip or knee replacement surgery: a randomized controlled trial. Arch Phys Med Rehabil. 2009;90(5):745-55.

36. Lee PG, Jackson EA, Richardson CR. Exercise prescriptions in older adults. Am Fam Physician. 2017;95(7):425-32.

37. Suetta C, Aagaard P, Rosted A, Jakobsen AK, Duus $B$, Kjaer M, et al. Training-induced changes in muscle CSA, muscle strength, EMG, and rate of force development in elderly subjects after long-term unilateral disuse. J Appl Physiol. 2004;97(5):1954-61.

38. Suetta C, Andersen JL, Dalgas U, Berget J, Koskinen SS, Aagaard P, et al. Resistance training induces qualitative changes in muscle morphology, muscle architecture, and muscle function in elderly postoperative patients. J Appl Physiol. 2008;105(1):180-86.

39. Winther SB, Foss OA, Husby OS, Wik TS, Klaksvik J, Husby VS. A randomized controlled trial on maximal strength training in 60 patients undergoing total hip arthroplasty: Implementing maximal strength training into clinical practice. Acta Orthop. 2018;89(3):295-01.

40. Fatouros IG, Kambas A, Katrabasas I, Leontsini D, Chatzinikolaou A, Jamurtas AZ, et al. Resistance training and detraining effects on flexibility performance in the elderly are intensity-dependent. J Strength Cond Res 2006;20(3) 634-42.

41. Husby VS, Helgerud J, Bjorgen S, Husby OS, Benum $P$, Hoff J. Early postoperative maximal strength training improves work efficiency $6-12$ months after osteoarthritis-induced total hip arthroplasty in patients younger than 60 years. Am J Phys Med Rehabil. 2010;89(4):304-14.
42. Unlu E, Eksioglu E, Aydog E, Aydoð ST, Atay G. The effect of exercise on hip muscle strength, gait speed and cadence in patients with total hip arthroplasty: a randomized controlled study. Clin Rehabil. 2007;21(8):706-11.

43. Nankaku M, Ikeguchi R, Goto K, So K, Kuroda Y, Matsuda S. Hip external rotator exercise contributes to improving physical functions in the early stage after total hip arthroplasty using an anterolateral approach: a randomized controlled trial. Disabil Rehabil. 2016;38(22):2178-83.

44. Mikkelsen LR, Mechlenburg I, Soballe K, Jorgensen LB, Mikkelsen S, Bandholm T, et al. Effect of early supervised progressive resistance training compared to unsupervised home-based exercise after fasttrack total hip replacement applied to patients with preoperative functional limitations: a single-blinded randomised controlled trial. Osteoarthr Cartil. 2014;22(12):2051-8.

45. Fisher J, Van-Dongen M, Sutherland R. Combined isometric and vibration training does not enhance strength beyond that of isometric training alone. J Sports Med Phys Fitness. 2015;55(9):899-904.

46. Turner AJ, Chander H, Knight AC. Falls in geriatric populations and hydrotherapy as an intervention: a brief review. Geriatrics. 2018;3(4):71.

47. Kutzner I, Richter A, Gordt K, Dymke, Damm $P$, Duda $G$, et al. Does aquatic exercise reduce hip and knee joint loading? In vivo load measurements with instrumented implants. PLoS ONE. 2017;12(3):e0171972 [9 p.].

48. Hauger AV, Reiman MP, Bjordal JM, Sheets C, Ledbetter L, Goode AP. Neuromuscular electrical stimulation is effective in strengthening the quadriceps muscle after anterior cruciate ligament surgery. Knee Surg Sports Traumatol Arthrosc 2018;26(2)399-410.

49. Kuru Çolak T, Kavlak B, Aydoğdu O, Sahin E, Açar $\mathrm{G}$, Demirbukem I, et al. The effects of therapeutic exercises on pain, muscle strength, functional capacity, balance and hemodynamic parameters in knee osteoarthritis patients: a randomized controlled study of supervised versus home exercises. Rheumatol Int. 2017;37(3):399-407.

50. Matheis C, Stöggl T. Strength and mobilization training within the first week following total hip arthroplasty. J Bodyw Mov Ther. 2018;22(2):519-27. 